\title{
Timed reactions to an object in apparent motion: Evidence on Cartesian and non-Cartesian perceptual hypotheses
}

\author{
NELSON COWAN and ELLYN GREENSPAHN \\ University of Missouri, Columbia, Missouri
}

\begin{abstract}
In three experiments on apparent motion, subjects were to press a computer key as quickly as possible either when the apparently moving stimulus passed the midpoint of its trajectory or when it reached the endpoint. If motion is perceived as a trajectory through a series of spatial locations, and if this type of percept is the basis of deliberate responding (assumptions that can be considered Cartesian in origin), then reaction times should be faster to the midpoint than to the endpoint. In contrast to these assumptions, there was no difference between reaction times to these two points. Alternative accounts of the results are discussed.
\end{abstract}

In apparent motion or "phi movement" (e.g., Kahneman \& Wolman, 1970; Kolers \& von Grünau, 1976; Ohtani, Ejima, \& Nishida, 1991; Wertheimer, 1912), an object that abruptly disappears from the visual field at some location, $A$, and abruptly reappears shortly afterward at a slightly different location, $B$, often gives rise to the illusory perception of movement of the object from location $A$ to location $B$. There are interesting riddles about apparent motion that remain to be solved. It is not known how, or when in the course of information processing, the phenomenal experience of motion is constructed. Nor is it clear how the experience of apparent motion affects the processing of information about the component stimulus objects. These questions are highlighted by a paradox. If apparent motion is assumed to traverse from location $A$ to location $B$, then the object should appear at any intermediate point, $i$, before it appears at location $B$. However, the motion logically cannot be perceived until after a stimulus has been presented at location $B$. Therefore, there must be an effect of information presented at location $B$ on the perception of earlier points in the trajectory, which has been described as a backward referral of information (Libet, 1981). ${ }^{1}$

To understand the nature of apparent motion, it may help to consider a task in which the subject is to press a key as quickly as possible, either when the perceived motion appears to complete its trajectory or when the perceived motion appears to reach a point in the middle of its trajectory. This is the task that we will investigate. On the basis of very different views of how perception fits into information processing, cases could be made that the reaction time (RT) should be faster to the intermediate point

This work was supported by NIH Grant HD-21338. We thank Tim Keller for technical assistance. Correspondence should be addressed to N. Cowan, Department of Psychology, 210 McAlester Hall, University of Missouri, Columbia, MO 65211 (e-mail: psycowan@mizzoul.missouri.edu). (i), faster to the endpoint $(B)$, or comparable for the two points. Let us consider these possible outcomes in turn.

\section{Faster Responding to the Intermediate Point: A Cartesian-Theater View}

Dennett and Kinsbourne (1992) described what they termed a Cartesian-theater view of perception, which was based on the writings of Descartes (1664/1985). In this view, in order for information from the sense organs to reach conscious awareness, it must be projected to one particular brain system, or "theater," of consciousness. Descartes assumed that theater resides in the pineal gland, an assumption that now appears naive even though the true anatomical basis of awareness remains uncertain. Without specifying where the neural seat of consciousness actually might be, predictions still can be made from a Cartesian-theater view, by incorporating assumptions from recent works conforming to fundamentally the same view.

A basic prediction can be derived as follows. Assume that information available to conscious awareness is used to form a conscious percept, and that the percept is used to make deliberate responses to the stimuli. Assume also that subjects see the apparently moving object pass the midpoint of the trajectory before it reaches the endpoint. It follows that they also should be able to press a response key sooner for the midpoint event than for the endpoint event.

Baars (1988) described a theory that might well be classified as a Cartesian-theater view, in which consciousness is seen as a "global workspace." Any chunks of information that reside in the workspace concurrently become associated with one another and available throughout the processing system. The workspace is said to be not only the seat of conscious awareness and experience but also the source of deliberate or voluntary responding. Thus, if the subject were asked to make a speeded response to one point in the trajectory of apparent motion, the re- 
sponse would have to be made on the basis of the same information that leads to the conscious percept, and in a manner consistent with that percept.

The reason that conscious percepts and deliberate responses should be consistent according to the model is that there is a presumed association between elements in the workspace. The workspace is thought to act as an integrator and interpreter that combines separate chunks of information and organizes them into a coherent, rational picture upon which deliberate actions are based. Some indirect support for this view of the normal conscious mind as an integrator and interpreter comes from research by Gazzaniga (1988) on patients with surgically separated left and right cerebral hemispheres. The left, verbal hemisphere appears to make up post hoc rationales so as to explain logically some actions instigated by the right hemisphere, the origin and purpose of which are unknown to the left hemisphere.

To reiterate, according to one modern version of the Cartesian-theater concept, what appears to occur sooner (presumably, passage of point $i$ ) should be responded to sooner. For this prediction to hold, it need not be assumed that the apparent rate of travel remains constant from point $A$ to point $B$; the prediction still could hold if there were apparent acceleration or deceleration within the trajectory. On the other hand, the prediction would not hold if the travel from the intermediate point $(i)$ to the endpoint $(B)$ appeared to be instantaneous. It has in fact been found that an interstimulus interval (ISI) of $0 \mathrm{msec}$ can give rise to apparent motion (Kahneman \& Wolman, 1970; Ohtani et al., 1991). However, there is good reason to believe that, even in the case of a $0-\mathrm{msec}$ ISI, the perceived rate of travel is not infinitely high. There is considerable evidence that apparent motion follows a path that is ecologically plausible given the constraints of physics as experienced by the subject (e.g., Shepard, 1984). Clearly, subjects are incapable of experiencing actual motion beyond a certain speed (Nakayama, 1985). For example, when a flashlight is switched on, the progression of the light beam across space clearly is too quick to be perceived. Similarly, if apparent motion is observed, it is likely to be experienced as noninstantaneous even if the ISI is $0 \mathrm{msec}$.

It is important to note that several versions of the Cartesian-theater view, making opposing predictions, are possible. The prediction described above is consistent with what Dennett and Kinsbourne (1992) called the Stalinesque version of the Cartesian-theater view, alluding to the withholding of information from awareness. Applied to the present task, it would suggest that when an endpoint stimulus at $B$ is first detected by some unconscious processing mechanism, the information that an object is present there is known at some point in the brain but is withheld from the conscious record until after a left-to-right movement sequence from $A$ to $B$ can be inserted. Another possible version of the Cartesiantheater view also leads to the same prediction, but was not acknowledged by Dennett and Kinsbourne. It states that the processing of the object at the endpoint location
$(B)$ is retarded by the two-stimulus context, so that the information that there is something at this endpoint is not available anywhere in the brain until after the information that something is moving from $A$ to $B$.

A second version of the Cartesian-theater view described by Dennett and Kinsbourne (1992) leads to a different prediction for the present task. It is termed Orwellian, alluding to the revision of information in awareness. In the present task, the presence of a stationary item at the right-hand side of the display could be registered in awareness initially, but quickly forgotten when it is replaced by the perception of movement from left to right. This view can be attributed to Goodman (1978, p. 83), who noted that the appearance of movement past a midpoint "seems to leave us a choice between a retrospective construction theory and a belief in clairvoyance." If the short-lived conscious percept of a stationary object at $B$ were enough to produce a speeded reaction even though this percept was forgotten soon afterward, then subjects should be able to respond to the endpoint faster than to the midpoint of the trajectory. However, it seems somewhat implausible that an attended event reaching awareness would be forgotten so quickly. Previous research has indicated that even brief shifts of awareness, as measured by errors in repetition or "shadowing" of a speech channel in selective listening tasks, produce stable memories of the briefly attended information (Cowan, Lichty, \& Grove, 1990; Wood \& Cowan, 1995). A non-Cartesian view described by Dennett and Kinsbourne can make the same predictions as the Orwellian version of the Cartesian-theater view, but on more plausible grounds; so, for convenience, the Orwellian stance will not be given much attention in this article.

Theories of perception that can be termed nonCartesian do not hold that all deliberate and conscious activities must arise from the same coherent neural workspace or Cartesian theater. Two different nonCartesian views to be discussed-a multiple-drafts view and a general information-processing view - are capable of making different theoretical predictions for the task under consideration.

\section{Faster Responding to the Endpoint: A Multiple-Drafts View}

Dennett and Kinsbourne (1992) described a view that is opposed to the notion that there is a Cartesian theater of some sort. Instead, they proposed that the brain may contain separate and sometimes conflicting descriptions of stimulus events simultanẹously. Conscious awareness was said to reflect the brain's attempt to weave a coherent story from the various perceptual strands, so that a perceptual event that was incoherent with the overall story might eventually be deleted from the story and therefore inaccessible to verbal report (i.e., it would remain outside of conscious awareness). In an analogy with creative writing, each perceptual event was termed a perceptual draft, with the perceptual events corresponding to the subject's subjective report forming the final draft. 
Dennett and Kinsbourne (1992, p. 194) stated that the "Multiple Drafts model agrees with [Goodman's, 1978, 'Orwellian' approach] that retrospectively the brain creates the content (the judgment) that there was intervening motion, and this content is then available to govern activity and leave its mark in memory." They go on to differentiate their view from that of Goodman in that they do not agree that the information must be projected to an area of consciousness where it must replace the previous information.

There is considerable flexibility in how the theory might be applied; it is not tightly specified. However, for the task under consideration, one plausible scenario is as follows. First, the brain would perceive separate objects at point $A$ and point $B$. Then, in a subsequent perceptual draft, the perceptual story would include only a single object that moved from $A$ to $B$. Assume that it takes time to form each perceptual draft, and that any perceptual draft can serve as the basis of an overt speeded reaction. The prediction is then that the RT to point $B$, based on a nonfinal perceptual draft, could be faster than the RT to the intermediate point $i$, which can be based only on the subsequent perceptual draft incorporating motion.

\section{No Difference Between Locations: Other Non-Cartesian Views}

In contrast to the above prediction, no difference between RTs to points $i$ and $B$ would be predicted by the multiple-drafts view if the formation of successive perceptual drafts was extremely rapid, or if a speeded reaction for some reason could not be based on the nonfinal draft. The prediction still would differ from that of the Cartesian-theater approach, in which the sequence of RTs must conform to a sequence of perceived locations.

In another non-Cartesian view that has been associated with the information-processing approach to cognition and perception (e.g., Massaro \& Cowan, 1993), it is believed that much processing takes place automatically and does not depend upon conscious awareness. In fact, according to this view, responses could be made on the basis of information different from the information that results in conscious perception.

One simple account of apparent motion from an information-processing approach is suggested by the theory of Kahneman and Wolman (1970). They proposed that each stimulus gives rise to an internal neural response. Apparent motion was said to be perceived only if the neural responses to the two stimuli "overlap." Although this theory might be in need of further clarification, it can be used to make some simple predictions. In the apparent motion situation, overlap between the neural responses would, of course, begin at the same time that the neural response to the second stimulus begins. It therefore is plausible that information about motion (the overlap between responses) could be available for an overt speeded response at the same time that information about the presence of a stimulus at location $B$ is available. This could result in no difference in RTs to the intermediate point and the endpoint of apparent motion.
(The conscious perception of motion nevertheless might depend on another stage of processing that interprets the meaning of the overlap in neural responses. Conceivably, the interpretation might not even be completed until after the speeded response is made, in direct contradiction to a Cartesian-theater view.)

There is one more consideration that could be important for the information-processing view described above. There might be a primitive stage of motion detection that is used to make a response in the proposed experimental situation and that is in some way available to consciousness. A logically coherent cognitive understanding of the display might still be incomplete at the time that the response is made.

There is considerable evidence for a primitive type of motion-detection mechanism in the brain that is independent of the perception of spatial location (for a review, see Nakayama, 1985). For example, in motion aftereffects such as the waterfall illusion, movement is perceived without any change in the perceived location (Wohlgemuth, 1911). According to this type of mechanism, it would be possible to detect motion consciously, yet without simultaneously being aware of a trajectory along the points involved in the motion. As soon as this motion-detection mechanism is set into play, the subject might respond by inferring that the midpoint was passed and the endpoint reached.

Presumably, in order to reach a rational understanding of the display in light of the subject's world knowledge, the subject at some point also would have to reach the perception or inference that the motion passed along a trajectory from one location to another. This more cognitive perceptual stage might reflect the second of two apparent motion processes that have been proposed previously (see Cavanagh \& Mather, 1989; Petersik, 1989). Petersik described this second stage, relative to the first stage, as more flexible and penetrable by cognitive processes. Given a two-stage description of motion perception, one information-processing view would state that the initiation of rapid responding need not await the operation of the second stage.

It could reasonably be claimed that such a two-stage description of motion perception also would qualify as one version of the multiple-drafts view, with the two stages of motion detection serving as the two drafts (though Dennett and Kinsbourne did not describe apparent motion in this way). In contrast, it would seem less likely that the two-stage description could be accepted within a Cartesian view. Descartes (1637/1965) characterized the visual system of the brain as a natural device that carried out mathematical geometric calculations automatically and intuitively, and that type of mental system would seem to perceive motion in terms of the change in spatial coordinates of the object. Thus, in the proposed experimental situation, we wish to assert simply that the prediction of no difference between midpoint and endpoint reaction times can be made by various non-Cartesian views, but not easily by a standard Cartesian view. 
Although the predictions we have discussed depend on special assumptions, and other variations are possible, they do serve to illustrate that a study of timed reactions to stimuli in apparent motion can be useful in constraining theories of perception. Accordingly, in the present article, we report on three experiments in which subjects were to respond rapidly, either when the object appeared to pass the spatial midpoint in an apparent motion trajectory or when it appeared to reach the endpoint of the trajectory.

\section{EXPERIMENT 1}

\section{Method}

Subjects. The subjects were 31 introductory psychology students who received course credit for their participation. One subject was excluded from the final sample for making too many false alarms (15) during the test session, leaving a final sample of 30.

Apparatus, Stimuli, and Procedure. Stimuli were displayed, and responses collected, on an IBM AT computer at a luminance adjusted to the subject's comfort, with green characters on a black background in a dimly lit room. Each trial began with the words GET READY presented both above and below the area to be taken up subsequently by a three-row display. After $1 \mathrm{sec}$, the ready signal disappeared and was replaced by the display, which covered an area on the screen $2.7 \mathrm{~cm}$ wide and $1.5 \mathrm{~cm}$ tall in the center of the screen. At the typical viewing distance of $50 \mathrm{~cm}$, the display subtended a visual angle of $3.1^{\circ}$ in width and $1.7^{\circ}$ in height.

The session began with two practice sessions, each of which continued until 10 experimental trials were run, half with a midpoint "*" marker and the other half with a right-hand, or endpoint, "*” marker. On each trial, the top row of the display consisted of three repetitions of the character "!" $(0.5 \mathrm{~cm}$ tall $)$ at the left, middle, and right of the $2.7-\mathrm{cm}$-wide display, which remained throughout the trial as reference points. In the middle row, at the same time, the character "*" $(0.25 \mathrm{~cm}$ tall $)$ appeared directly under either the middle or the right-hand "!", indicating the location at which the subject was to respond as the moving character arrived. In the third row, at the same time, an "o" $(0.25 \mathrm{~cm}$ tall $)$ appeared directly under the left-hand asterisk. This was the character that eventually would appear to move. Initially, the display for a respondat-midpoint trial, for example, would look about as follows:

$$
\begin{array}{lll}
1 & !
\end{array}
$$

After a random interval varying from 1 to $2 \mathrm{sec}$, the "o" disappeared. On half of the trials, $100 \mathrm{msec}$ after the " $\mathrm{o}$ " disappeared, the same character appeared directly under the right-hand "!" character, giving rise to apparent motion of the " 0 " (thus, ISI = $100 \mathrm{msec}$ ). The subject's task was to press the enter key of the computer as quickly as possible after the "o" appeared to get as far as the "*" marker. This display remained on until the subject responded to it, which ended the trial.

On the other half of the trials, again after $100 \mathrm{msec}$, the " 0 " instead reappeared under the left-hand "!". This procedure was used so that the subjects would not be able to anticipate movement on the basis of the disappearance of the left-hand " 0 ". Whenever they made a response to an "o" that had reappeared at the left instead of shifting to the right-hand side of the display, that response was counted as a false alarm. If an " 0 " reappeared at the left-hand side of the display and the subject did not make the mistake of responding to it, then the trial ended $1 \mathrm{sec}$ after the reappearance. ${ }^{2}$

For all types of trial, the end of one trial was followed immediately by the ready signal for the next trial to begin. The probability that the current trial would be a control trial in which there was no shift to the right always was .5. However, the location of the "*" marker was maintained across trials until by chance the "o" did shift to the right (i.e., an experimental trial). The sequence of "*" marker locations in experimental trials was random, with the restriction that there could be no more than two successive experimental trials with the same marker location.

After the practice session, the test session began, and it continued until a total of 100 experimental trials were run, again half with a midpoint "**" marker and the other half with an endpoint "**" marker. When the necessary number of trials in one condition was reached, only the other condition was run (it could be either condition). Given the random determination of trial types, each subject also received about 100 catch trials in which the "o" disappeared but then reappeared in the same location, about half of which occurred with midpoint markers and half with endpoint markers.

\section{Results}

None of the subjects made more than five false alarms during the test session, except one subject who was excluded from the final sample as noted above. For the other subjects, the mean number of false alarms for the test session was $1.27(S D=1.50)$. RT scores were calculated from the appearance of the circle at the endpoint and the subject's response to the movement, and RTs larger than $1 \mathrm{sec}$ were disqualified because they were assumed to reflect attentional lapses. This occurred only rarely, in $0.4 \%$ of the midpoint trials and $0.7 \%$ of the endpoint trials. Mean RTs for each subject in both experimental conditions were calculated.

The averages of these individual subject means were $338.11 \mathrm{msec}(S D=41.88, S E M=7.65)$ for trials in which the marker was at the midpoint and an almost identical $338.50 \mathrm{msec}(S D=44.36, S E M=8.10)$ for trials in which the marker was at the endpoint. In a withinsubject analysis of variance (ANOVA) of RTs, the difference between conditions did not approach significance $\left[F(1,29)<1, M S_{\mathrm{e}}=163.69\right]$.

Most of the variance in RTs was between subjects, and most subjects yielded similar mean RTs for the midpoint and endpoint conditions. This can be observed clearly within (endpoint-midpoint) difference scores $(M=$ $0.39 \mathrm{msec}$ ), which had a $95 \%$ confidence interval of only $6.76 \mathrm{msec}$. Given the large number of trials per subject, even individual subject means were fairly reliable. The average individual's $S E M$ was $10.91 \mathrm{msec}$ in the midpoint condition and $11.09 \mathrm{msec}$ in the endpoint conditions.

The absence of a significant difference between conditions cannot easily be attributed to a lack of power of the experiment. A power analysis (Buchner, Faul, \& Erdfelder, 1992) indicated that a difference between means as small as $20 \mathrm{msec}$ could have been detected with a power $(1-\beta)$ of about .95 .

One potential problem with the experimental design is that it was not completely balanced across the session. The randomization permitted no more than 2 trials of a particular type in succession until all trials of one type were presented, but then all remaining trials of the other type were presented. This resulted in a final run of, on the average, 4.16 trials of one type $(S D=2.77)$, as op- 
posed to a limit of 2 trials elsewhere in the session. It is conceivable that this lack of balance in the design was a problem because of effects of practice or fatigue. However, to take into account the effect of the final runs, RT means were recalculated, with all but the first 2 trials of the final runs omitted. The RT means calculated in this way $(338.02 \mathrm{msec}$ for the midpoint and $338.16 \mathrm{msec}$ for the endpoint) were nearly the same as before, and again the effect of condition failed to approach significance $\left[F(1,29)<1, M S_{\mathrm{e}}=152.05\right]$. In the subsequent experiments, the designs were completely balanced.

\section{Discussion}

The present finding appears to rule out the possibility that the phenomenal experience of motion from one discrete point to another underlies the RTs. If it were the basis of RTs, one would expect the RT to a midpoint marker to be faster than the RT to an endpoint marker.

Before this conclusion can be accepted, however, another possibility must be ruled out. The subjects might have perceived the "o" stimulus as passing the midpoint considerably sooner than the endpoint, but their RTs still might not reflect this because of the time it takes to mobilize the motor system. For example, suppose that the subjects perceived the midpoint target, on the average, $120 \mathrm{msec}$ after the left-hand "o" stimulus disappeared, and that they perceived the endpoint target, on the average, $40 \mathrm{msec}$ later than the midpoint. Suppose further that they only reached a state of motor readiness $200 \mathrm{msec}$ after the left-hand "o" stimulus disappeared. Then their RTs could not reflect the difference in perception times. Experiment 2 was designed to examine this possibility by requiring a response to a stimulus presented earlier at the midpoint and to examine other details of apparent motion.

\section{EXPERIMENT 2}

To address the concern of the minimal RT, on some trials an actual "o" stimulus was briefly flashed at the midpoint of the phi movement trajectory, beginning at half of the $100-\mathrm{msec}$ ISI that elapsed between the beginning and endpoint stimuli. If subjects are incapable of making a response any more quickly than the responses that occurred to an endpoint stimulus in Experiment 1 , then this actual midpoint stimulus should make no difference.

In another condition, the midpoint stimulus was not flashed until $50 \mathrm{msec}$ after the endpoint stimulus. The effect was disconcerting. Because the midpoint stimulus disappeared quickly (whereas the endpoint stimulus remained, pending the subject's response), a movement from left to right still could be perceived, although some subjects thought that the timing of the stimulus was somehow awry. We included this condition for exploratory purposes, to find out whether the delayed midpoint stimulus could delay the subject's response to a midpoint marker by predominating over the earlier midpoint percept that is based only on apparent motion. If so, it would favor an
Orwellian hypothesis, in which fleeting conscious percepts can be amended in memory by subsequent information.

In all, two marker conditions (midpoint, endpoint) were crossed with three midpoint stimulus conditions $(-50 \mathrm{msec},+50 \mathrm{msec}$, none) for a total of six conditions. Figure 1 illustrates the timing of displays for the three conditions in which the "*" marker indicating the target location was placed in the middle of the display; the remaining three conditions were the same, except with the "*" marker located under the rightmost of the three "!" symbols in the display.

\section{Method}

Subjects. The subjects were 23 introductory psychology students who had not participated in Experiment 1 and who received course credit for their participation.

Method. The progression of events on each trial was the same as in Experiment 1, except that on some trials the "o" character was presented at the midpoint. On some of these trials $(-50-\mathrm{msec}$ condition), it began $50 \mathrm{msec}$ before the onset of the right-hand (endpoint) "o" and lasted for $50 \mathrm{msec}$, at which time the "o" at midpoint was extinguished and an "o" was presented at the righthand location. On other trials (+50-msec condition), the "o" was presented only $50 \mathrm{msec}$ after the onset of the endpoint stimulus, and again this midpoint stimulus lasted $50 \mathrm{msec}$. The right-hand "o," on the other hand, always persisted on the screen until the subject made a response, as in Experiment 1. The midpoint stimulus never was presented on catch trials, in which the "o" disappeared and then reappeared $100 \mathrm{msec}$ later at the beginning (right-hand) location. Thus, in all, there were six experimental conditions, formed by combining "*" markers to be responded to at two locations (midpoint, right) with three midpoint " $o$ " presentation conditions (no midpoint "o," as in Experiment 1; midpoint "o" $50 \mathrm{msec}$ before the right-hand "o" appears; midpoint "o" $50 \mathrm{msec}$ after the right-hand "o" appears).

There were two practice sessions with 12 experimental trials each ( 2 of each of the 6 conditions), and then a test session with 120 experimental trials ( 20 of each condition). Within the test session, each successive block of 6 experimental trials included each trial type once. As in Experiment 1, the control trials occurred randomly with a .5 probability on each trial. In all other ways, the method was the same as that in Experiment 1.

\section{Results}

As in Experiment 1, the frequency of fdse alarms was fairly low $(M=2.83, S D=3.30)$. Also as in Experiment 1, RTs longer than $1 \mathrm{sec}$ were omitted in the calculation of subject means. The percentage of trials in which this occurred ranged among the six conditions from $0.7 \%$ to $1.1 \%$ of the trials. The mean RTs for each condition, averaged across individual subject means, are shown in Table 1. The table indicates that the means for the -50 -msec condition were about $50 \mathrm{msec}$ below the means for the other two conditions.

The RT means were entered into an ANOVA with two within-subject factors: the location of the "*" marker (midpoint versus right-hand side) and the timing of the stimulus at the midpoint location $(-50 \mathrm{msec},+50 \mathrm{msec}$, none). Consistent with Table 1 , this analysis yielded only an effect of the stimulus at midpoint $[F(2,44)=$ 75.79, $\left.M S_{\mathrm{e}}=447.95, p<.001\right]$. Post hoc pairwise Tukey tests revealed that the $-50-\mathrm{msec}$ condition mean was significantly lower than both the $+50-\mathrm{msec}$ and the no- 


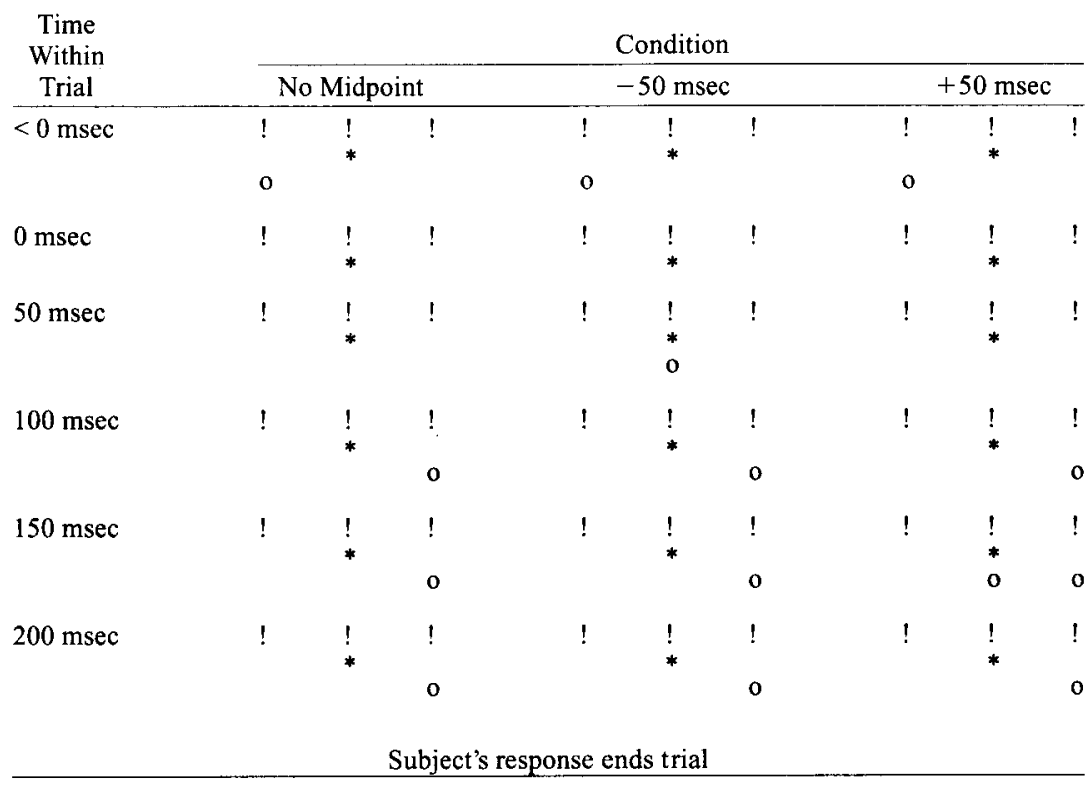

\begin{abstract}
Figure 1. An illustration of the display as it appeared at various times within a trial, for three midpoint "o" presentation conditions of Experiment 2. The conditions shown are for a midpoint target location, as indicated by the location of the " $*$ " marker. The subjects were to respond to movement of the " 0 " to or past the marker location. The conditions for the endpoint target location (not shown) were the same, except that the "**" marker was located under the right-hand "!" marker. -50 msec and +50 msec represent the midpoint "o" stimulus onset times relative to the onset of the right-hand "o" stimulus.
\end{abstract}

midpoint-"o" means ( $p$ s $<.01$ ), but that the difference between the +50 -msec and no-midpoint conditions did not approach significance. Thus, having an actual stimulus at the midpoint $50 \mathrm{msec}$ before the endpoint stimulus hastened responses to either the midpoint or the endpoint.

Consistent with Experiment 1, the effects involving the location of the "**" marker did not approach significance. In the conditions with no stimulus at the midpoint, the difference in RTs for the endpoint and midpoint target locations $(M=-2.87 \mathrm{msec})$ had a $95 \%$ confidence interval of $12.26 \mathrm{msec}$.

\section{Discussion}

These results provide reassurance that the absence of an effect of the marker location in the case of apparent motion cannot be attributed to an insensitivity of the RT measure, given that the RT was altered by a real midpoint stimulus presented before the endpoint stimulus. The means are what one would expect if subjects use each piece of relevant information as soon as it is presented. Moreover, responses were not delayed by later information in the case of the $+50-\mathrm{msec}$ condition.

There was, however, one surprising outcome of this experiment. Placing an " $\mathrm{o}$ " stimulus at the midpoint $50 \mathrm{msec}$ before the endpoint "o" stimulus not only speeded the response to a midpoint marker but also speeded the response to an endpoint marker by almost the same amount (see Table 1). There are two possible accounts of this effect. One account states that the midpoint presentation helped the subject to perceive the motion associated with the endpoint "o" presentation more quickly than otherwise would have been possible. Another account, however, states simply that the subject did not wait for apparent motion, given that the midpoint "o" was presented. Whenever the midpoint "o" occurred, it was followed $50 \mathrm{msec}$ later by an endpoint "o," so the subjects might have anticipated the occurrence of the endpoint "o." Experiment 3 was conducted to clear up this ambiguity. It had the same design as Experiment 2,

Table 1

Mean Response Times and Standard Errors of the Means (in Milliseconds) for Each Condition in Experiment 2

\begin{tabular}{|c|c|c|c|c|c|c|}
\hline \multirow[b]{3}{*}{ Marker Position } & \multicolumn{6}{|c|}{ Midpoint "o" Stimulus } \\
\hline & \multicolumn{2}{|c|}{$-50 \mathrm{msec}$} & \multicolumn{2}{|c|}{$+50 \mathrm{msec}$} & \multicolumn{2}{|c|}{ Absent } \\
\hline & $M$ & $S E M$ & $M$ & $S E M$ & $M$ & $S E M$ \\
\hline Midpoint & 294.41 & 9.48 & 346.25 & 10.71 & 347.49 & 11.07 \\
\hline Endpoint (Right) & 305.64 & 10.11 & 349.83 & 11.51 & 344.62 & 10.75 \\
\hline
\end{tabular}

Note- $-50 \mathrm{msec}$ and $+50 \mathrm{msec}$ are the onset times of the midpoint "o" stimulus relative to the onset of the right-hand "o" stimulus. 
except that a second type of catch trial was added, in which the "o" stimulus progressed to the midpoint but then failed to progress further (to the endpoint).

\section{EXPERIMENT 3}

\section{Method}

Subjects. The subjects were 24 students who had not participated in the first two experiments.

Apparatus, Stimuli, and Procedure. In place of the IBM AT computers used in the previous experiments, all but 5 subjects in this experiment viewed the stimuli on newer IBM-compatible computers using Intel 80486 microprocessors. The monitor display for these computers was slightly larger than before; the width of the stimulus display (which, in Experiments 1 and 2, was 2.7 $\mathrm{cm}$ ) was now $3.0 \mathrm{~cm}$, which, at a typical viewing distance of 50 $\mathrm{cm}$, corresponded to a visual angle of $3.4^{\circ}$. The display was now white (instead of green) on a black background. No systematic differences between the responses of the subjects using the older versus the newer equipment could be observed.

The procedure was the same as in Experiment 2, with one exception. On trials in which the "*" marker was at the endpoint and the "o" stimulus was presented at the midpoint before the endpoint stimulus ( -50 -msec condition), after the midpoint " 0 " disappeared the probability of it reappearing at the endpoint was only .50. The other half of the time, the " $o$ " reappeared at the midpoint, constituting a new type of catch trial. Given that the "*" marker on these trials indicated the endpoint as the target location, responses to the " $o$ " reappearing at the midpoint were incorrect and the subject received a computer message warning of the mistake. Thus, there were two types of catch trials in this experiment, corresponding to an "o" stimulus disappearing and reappearing either at the beginning of the trajectory, for either target location (as in the previous experiments), or at the middle of the trajectory, for endpoint target locations only.

\section{Results}

The subjects made an average of only 1.83 false alarms $(S D=2.88)$ to the catch trials at the beginning of the apparent motion trajectory. False alarms at the midpoint were more frequent but still fairly low $(M=2.92$, $S D=2.52$ ). As in Experiments 1 and 2, RTs greater than $1 \mathrm{sec}$ were omitted from the analysis of responses on experimental trials. The proportion of such trials was low, ranging from $0.2 \%$ to $0.8 \%$ of all trials in particular conditions.

The mean RTs for experimental trials are shown in Table 2. The corresponding data were entered into an ANOVA with the same factors as used in Experiment 2. There was again a main effect of the midpoint presentation condition $\left[F(2,46)=36.32, M S_{\mathrm{e}}=392.46, p<.001\right]$. As in Experiment 2, post hoc Tukey pairwise compar- isons indicated that the -50 -msec midpoint-presentation condition mean was significantly lower than both the +50 -msec and the no-midpoint-presentation means ( $p$ s $<$ .01 ), though the difference between the latter two conditions did not approach significance. Thus, the inclusion of frequent midpoint catch trials in Experiment 3 did not fundamentally change the effect of midpoint presentation.

Consistent with both of the previous experiments, the main effect of the location of the "*" marker did not approach significance. In the conditions with no stimulus at the midpoint, the difference in RTs for the endpoint and midpoint target locations $(M=-12.63 \mathrm{msec})$ had a $95 \%$ confidence interval of $14.11 \mathrm{msec}$.

On the other hand, in Experiment 3, the interaction of the midpoint presentation with the target location reached significance $\left[F(2,46)=4.29, M S_{\mathrm{e}}=408.19, p<\right.$ $.02]$. It appears from Table 2 that the effect of midpoint presentation was smaller when the target location was the endpoint than when it was the midpoint. Nevertheless, separate analyses for the midpoint target location and the endpoint target location both produced strong main effects of the midpoint presentation $(p s<.001)$.

One clue to the nature of the interaction is provided by separate analyses for each midpoint presentation. When there was no midpoint presentation, responses were marginally faster to the endpoint than to the midpoint $\left[F(1,23)=4.33, M S_{\mathrm{e}}=558.07, p<.08\right]$. On the other hand, in the -50 -msec midpoint-presentation condition, responses were marginally slower to the endpoint than to the midpoint $\left[F(1,23)=3.17, M S_{\mathrm{e}}=444.89, p<\right.$ $.09]$. The difference for the +50 -msec condition did not approach significance.

\section{Discussion}

The main finding of Experiment 2, an RT advantage of receiving an "o" stimulus at the midpoint of the display $50 \mathrm{msec}$ before the endpoint "o" stimulus, was replicated in Experiment 3. On the other hand, the advantage was about $50 \mathrm{msec}$ in Experiment 2, as opposed to about $30 \mathrm{msec}$ in Experiment 3 . Given the presence of an extra type of catch trial in Experiment 3, this more moderate advantage is probably more meaningful psychologically.

This result is of use in interpreting the main finding from all three experiments that, in the absence of an actual midpoint stimulus, subjects respond to apparent movement past the midpoint of the display and apparent arrival at the endpoint in comparable amounts of time. If

Table 2

Mean Response Times and Standard Errors of the Means (in Milliseconds) for Each Condition in Experiment 3

\begin{tabular}{|c|c|c|c|c|c|c|}
\hline \multirow[b]{3}{*}{ Marker Position } & \multicolumn{6}{|c|}{ Midpoint "o" Stimulus } \\
\hline & \multicolumn{2}{|c|}{$-50 \mathrm{msec}$} & \multicolumn{2}{|c|}{$+50 \mathrm{msec}$} & \multicolumn{2}{|c|}{ Absent } \\
\hline & $M$ & $S E M$ & $M$ & $S E M$ & $M$ & $S E M$ \\
\hline Midpoint & 296.13 & 15.26 & 334.67 & 16.65 & 337.38 & 14.99 \\
\hline Endpoint (Right) & 306.96 & 12.59 & 328.75 & 11.84 & 324.75 & 12.63 \\
\hline
\end{tabular}

Note- $-50 \mathrm{msec}$ and $+50 \mathrm{msec}$ are the onset times of the midpoint " 0 " stimulus relative to the onset of the right-hand "o" stimulus. 
the subjects actually perceived motion past the midpoint at least $30 \mathrm{msec}$ before they perceived motion to the endpoint, but simply could not get their motor systems to respond to the midpoint stimuli quickly enough to reflect this priority, then they should not have been able to respond to a real stimulus at the midpoint $30 \mathrm{msec}$ more quickly. Thus, it appears that perception of the movement past the midpoint and perception of movement to the endpoint in apparent motion are constructed in the brain simultaneously or nearly so, even though the perception is one involving apparent temporal sequence.

The addition of frequent catch trials in which the "o" remained at the midpoint rather than progressing to the endpoint did not alter the fact that the midpoint presentation speeded responses to the endpoint target location as well as to the midpoint location. The midpoint presentation thus appears to have served to prime the perception of movement based on the appearance of the endpoint stimulus. The advantage of this priming was impressive-in fact, only marginally less than the advantage of a midpoint presentation for responding to movement past the midpoint (see Table 2).

Finally, with no midpoint presentation, in Experiment 3 the response to the endpoint (about $325 \mathrm{msec}$ ) was marginally faster than the response to apparent motion past the midpoint (about $337 \mathrm{msec}$ ), as shown in Table 2. It is unwise to place much stock in a marginal effect, but if this effect turned out to be real, it would be consistent with the multiple-drafts model of perception described above. In any case, this provides even stronger evidence that the Cartesian-theater view, as described above, is wrong. In that view, the RT difference should be in the other direction, conforming to a sequence in which movement of the "o" stimulus past the midpoint is perceived to have occurred before its arrival at the endpoint.

\section{GENERAL DISCUSSION}

This study contrasts several basic views of perception and the predictions they make in a simple task based on stimuli that give rise to the perception of apparent motion. The subjects were to press a button as quickly as possible when the target object appeared to reach either the midpoint or the endpoint of its apparent motion trajectory. If this task were accomplished on the basis of the perceptual events in a manner that reflected the relative times at which they were experienced, as it should be according to one common view (a version of the Cartesiantheater view, discussed in the introduction), and if the appearance of the object passing the midpoint preceded the appearance of the object reaching the endpoint, then the mean RT to the midpoint should have been faster than the mean RT to the endpoint. Instead, it was found in three experiments that the midpoint and endpoint RTs were nearly identical.

One never can state with certainty that two means are identical, but several factors strengthen the contention that the RTs to the midpoint and endpoint target locations really were very similar. First, the means were quite similar in these two conditions. For trials with no actual midpoint stimulus, the difference in means was approximately 0,3 , and $13 \mathrm{msec}$ in the three experiments. (In the marginally significant $13-\mathrm{msec}$ difference, responding was slower to the midpoint stimulus, in opposition to the Cartesian-theater view described above.) Second, a statistical power analysis indicated that it should have been possible to detect an RT difference as small as $20 \mathrm{msec}$ with a power of .95 . Third, the fact that significantly faster RTs were observed when an actual stimulus was presented at the midpoint $50 \mathrm{msec}$ before the occurrence of the endpoint stimulus (in Experiments 2 and 3) demonstrates that the RT measure was not simply insensitive to the precise timing of events in the display. Given the result of Experiment 3, there may be some possibility that the RT to the midpoint actually is slightly slower than the RT to the endpoint, but there is no evidence suggesting the opposite (that the RT to the midpoint is faster).

An additional ANOVA across all three experiments $(N=77)$ was conducted for the conditions with no midpoint presentation. Again, there was no difference between the mean for the RT to the midpoint target location $(M=340.68, S D=56.01)$ and the endpoint target location $(M=336.04, S D=52.37)[F(1,76)=2.29$, $\left.M S_{\mathrm{e}}=363.16, p>.1\right]$. All of this goes against the Cartesian-theater view described in the introduction.

As suggested in the introduction, the Cartesian-theater view can be salvaged if one is willing to make the Orwellian assumption described by Dennett and Kinsbourne (1992) - that is, the assumption that information about a stationary object at the endpoint can reach awareness but then be forgotten when it is quickly replaced by the perception of motion. An advantage for the endpoint is predicted if subjects can use the information about a stationary object at the endpoint to initiate a speeded response during the fleeting moment when the information is in awareness, before it is replaced by a perception of movement.

One possible prediction of the multiple-drafts model is similar to predictions of the Orwellian view. The image of a static object at $B$ arrives, and, subsequently, the perception of motion is constructed. (The only difference is that, in the perceptual-drafts view, both types of percept remain in the system rather than one being replaced by another.) For these models, the response to the endpoint in principle should precede the response to the midpoint. However, the advantage for the endpoint RT predicted by these views failed to reach significance in any of the experiments.

The Cartesian-theater view, as described above, embodied two assumptions. The first was that the observer forms a conscious percept in which the moving object reaches the midpoint of the trajectory and then subsequently reaches the endpoint of the trajectory. (As mentioned above, this assumption may be inferred from considerations described by Descartes, 1637/1965; see also Sedgwick, 1986.) The second was that responses are based on this conscious percept. (This assumption may 
be inferred from considerations described by Baars, 1988, Dennett \& Kinsbourne, 1992, and Descartes, $1664 / 1985)$. Given that RTs were the same for the midpoint and the endpoint markers, it appears that at least one of these assumptions is incorrect. It seems instead that there is a source of information about motion that precedes (or, conceivably, occurs instead of) a logical, spatially coherent percept, and that this source can be used to make deliberate responses. It is not yet clear whether the non-Cartesian outcome is most aptly viewed as a type of multiple-drafts mechanism or an informationprocessing mechanism with multiple processing stages.

Apparent movement to the endpoint of the display created by a right-hand "o" stimulus logically implied movement past the midpoint, so from this point of view it is not surprising that, in all three experiments, the RTs to the midpoint and endpoint were very similar. Moreover, when an actual midpoint "o" cue to movement was presented $50 \mathrm{msec}$ earlier (Experiments 2 and 3), RTs for both midpoint and endpoint target locations were faster as a result. The midpoint stimulus appears to have sped up the psychological interpretation of movement leading ultimately toward the endpoint stimulus.

Though the present data are unable to specify definitively what the mechanism of perception is, they do rule out at least one plausible class of perceptual theorynamely, the versions of the Cartesian-theater view in which speeded reactions to apparent motion are based directly on a coherent, conscious percept of movement from a beginning point, through the intermediate points, to an endpoint. The midpoint RT was not faster than the endpoint RT as it should have been if the chronology of speeded responses matched the chronology of perceptual events. The data are useful in illustrating how behavioral evidence can help to address basic philosophical questions about perception.

\section{REFERENCES}

BAARS, B. J. (1988). A cognitive theory of consciousness. Cambridge: Cambridge University Press.

Buchner, A., Faul, F., \& ERdFelder, E. (1992). G-Power: A priori, post-hoc, and compromise power analyses for the Macintosh [computer program]. Bonn: Bonn University.

Cavanagh, P., \& Mather, G. (1989). Motion: The long and the short of it. Spatial Vision, 4, 103-129.

Cowan, N., Lichty, W., \& Grove, T. R. (1990). Properties of memory for unattended spoken syllables. Journal of Experimental Psychology: Learning, Memory, \& Cognition, 16, 258-269.

Dennett, D. C., \& Kinsbourne, M. (1992). Time and the observer: The where and when of consciousness in the brain. Behavioral \& Brain Sciences, 15, 183-247.

DesCaRTES, R. (1965). Discourse on method, optics, geometry, and meteorology (P. Olscamp, Trans.). Indianapolis: Bobbs-Merrill. (Original work published 1637)

DESCARTES, R. (1985). Treatise on man. In J. Cottingham, R. Stoothoff, \& D. Murdoch (Eds. \& Trans.), The philosophical writings of
Descartes (Vol. 1, pp. 99-108). Cambridge: Cambridge University Press. (Original work published 1664)

GaZZANiga, M. S. (1988). Brain modularity: Towards a philosophy of conscious experience. In A. J. Marcel \& E. Bisiach (Eds.), Consciousness in contemporary science (pp. 218-238). Oxford: Oxford University Press, Clarendon Press.

Goodman, N. (1978). Ways of worldmaking. Hassocks, U.K.: Harvester.

Kahneman, D., \& Wolman, R. E. (1970). Stroboscopic motion: Effects of duration and interval. Perception \& Psychophysics, 8, 161164.

Kolers, P. A., \& von Grünau, M. (1976). Shape and color in apparent motion. Vision Research, 16, 329-335.

LIBET, B. (1981). Experimental evidence for subjective referral of sensory experience backwards in time: Reply to P. S. Churchland. Philosophy of Science, 48, 182-197.

Massaro, D. W., \& Cowan, N. (1993). Information processing models: Microscopes of the mind. Annual Review of Psychology, 44, 383-425.

Nakayama, K. (1985). Biological image motion processing: A review. Vision Research, 25, 625-660.

Ohtani, Y., EJima, Y., \& Nishida, S.-Y. (1991). Contribution of transient and sustained responses to the perception of apparent motion. Vision Research, 31, 999-1012.

Petersik, J. T. (1989). The two-process distinction in apparent motion. Psychological Bulletin, 106, 107-127.

SEDGwick, H. A. (1986). Space perception. In K. R. Boff, L. Kaufman, \& J. P. Thomas (Eds.), Handbook of perception and human performanc: Vol. 1. Sensory processes and perception (pp. 21-1 to 21-57). New York: Wiley.

SHEPARD, R. N. (1984). Ecological constraints on internal representations: Resonant kinematics of perceiving, imagining, thinking, and dreaming. Psychological Review, 91, 417-447.

WerTheimer, M. (1912). Experimentelle studien über das Sehen von Bewegung [Experimental studies of the seeing of movement] Zeitschrift für Psychologie, 61, 161-265.

WOHLGEMUTH, A. (1911). On the aftereffect of seen movement British Journal of Psychology Monograph Supplement, 1(1).

Woon, N., \& Cowan, N. (1995). The cocktail party phenomenon revisited: How frequent are attention shifts to one's name in an irrelevant auditory channel? Journal of Experimental Psychology: Learning, Memory, \& Cognition, 21, 255-260.

\section{NOTES}

1. Dennett and Kinsbourne (1992) suggested that the paradox is dramatized in the situation in which the object that appears to move is presented in one color at one location and a different color at the other location. In such a situation, Kolers and Grünau (1976) had subjects match the apparently moving object to an adjustable color standard at various points in the trajectory, and the results suggested an abrupt change from the first color to the second at about the middle of the apparent trajectory. We considered basing our experiments on this color phi phenomenon. However, when we programmed and observed the experimental setup, the target object actually appeared rather colorless during motion. We therefore wonder if the color matches that Kolers and Grünau obtained reflect primarily task demands and the limitation in available responses rather than a vividly experienced color transition.

2. Stimulus display times were approximate given that the display was not synchronized with the computer monitor's refresh rate of $60 \mathrm{~Hz}$.

(Manuscript received December 28, 1993; revision accepted for publication November 17, 1994.) 